\title{
Varieties Generated by Certain Models of Reversible Finite Automata
}

\author{
Marats Golovkins* Jean-Eric Pin
}

Received: December 18, 2007; published: June 21, 2010.

\begin{abstract}
Reversible finite automata with halting states (RFA) were first considered by Ambainis and Freivalds to facilitate the research of Kondacs-Watrous quantum finite automata. In this paper we consider some of the algebraic properties of RFA, namely the varieties these automata generate. Consequently, we obtain a characterization of the Boolean closure of the classes of languages recognized by these models.

We also obtain an equality which relates varieties of ordered $\mathcal{J}$-trivial monoids with the variety of $\mathcal{R}$-trivial monoids.
\end{abstract}

\section{Introduction}

In this paper we study reversible finite automata (RFA). Being entirely classical, the model is however a special case of Kondacs-Watrous quantum finite automata and was introduced in [5]. Quantum finite automata (QFA) are of a specific interest, since the family of these models represent finite memory real-time quantum mechanical devices. On the other hand, recently it has been demonstrated [3] that these models are worth studying also from the point of view of classical algebraic automata theory. The first models of QFA are due to [13] and [15]. Other models are proposed and studied, for example, in $[9,16,6,8,3,11,4]$, etc. In principle, the different types of QFA reflect the different ways how the results of computation can be interpreted, i.e., quantum measurements. By applying various restrictions, it is even possible to get deterministic and probabilistic special cases of QFA. Such models sometimes prove to be extremely useful in the research of the properties of QFA. For example, probabilistic reversible automata (PRA) [11] are instrumental to prove that Latvian QFA [3] recognize exactly the regular languages whose

\footnotetext{
*Supported by the Latvian Council of Science, grant No. 09.1437 and by the European Social Fund, contract No. 2009/0216/1DP/1.1.1.2.0/09/APIA/VIAA/044. 
syntactic monoids are block groups. Moreover, PRA and LQFA recognize the same class of regular languages.

In Section 2 we introduce the finite automata models discussed further in the paper. Section 3 recalls notation on varieties used in this paper. Section 4 deals with injective finite automata (IFA), which are in turn a special case of RFA. IFA are closely related to a deterministic special case of Brodsky-Pippenger QFA [9]. We give an exact characterization of languages which are recognized by IFA and conclude that the syntactic monoids of this class generate the variety of commuting idempotent monoids, ECom. In Section 5 we show that the syntactic monoids of the languages recognized by RFA generate $\mathbf{E R}_{\mathbf{1}}$, the variety defined by the identity $x^{\omega} y^{\omega} x^{\omega}=x^{\omega} y^{\omega}$. Section 6 specifies algebraic conditions for a language to be recognized by RFA or IFA. In Section 7 it is proved that semidirect products of ordered $\mathcal{J}$-trivial monoids of opposite order generate the variety of $\mathcal{R}$-trivial monoids.

A preliminary version of this paper appeared in [12].

\section{Preliminaries}

In this paper, by minimal automaton of a regular language we understand a complete minimal deterministic finite automaton recognizing the language (the transition function is defined for any state and any input letter). Two automata (deterministic or not) are said to be equivalent if they accept the same language. We denote by $L^{c}$ the complement of a language $L$. We do not recall the general definition for KondacsWatrous QFA, which can be found in [13]. The definition of RFA is obtained from Kondacs-Watrous QFA by adding the restriction that any transition is deterministic:

Definition 2.1. A reversible finite automaton $\mathcal{A}=\left(Q, \Sigma \cup\{\$\}, q_{0}, Q_{a}, Q_{r}, \cdot\right)$ is specified by a finite set of states $Q$, a finite input alphabet $\Sigma$, an end-marker $\$ \notin \Sigma$ and an initial state $q_{0} \in Q$. The set $Q$ is the union of two disjoint subsets $Q_{h}$ and $Q_{n}$, called the set of halting and non-halting states, respectively. Further, the set $Q_{h}$ is the union of two disjoint subsets $Q_{a}$ and $Q_{r}$ of $Q$, called the set of accepting and rejecting states, respectively. The transition function $(q, \sigma) \rightarrow q \cdot \sigma$ from $Q \times(\Sigma \cup\{\$\})$ into $Q$ satisfies the following conditions:

for all $\sigma \in \Sigma \cup\{\$\}, \quad q_{1} \cdot \sigma=q_{2} \cdot \sigma$ implies $q_{1}=q_{2}$;

if $q$ is non-halting, then $q \cdot \$$ is halting.

The first condition is equivalent to each letter $\sigma \in \Sigma \cup\{\$\}$ inducing a bijection on $Q$. A RFA reads any input word starting with the first letter. As soon as the automaton enters a halting state, the computation is halted and the word is either accepted or rejected, depending on whether the state is accepting or rejecting. The end-marker \$ insures that any input word followed by the end-marker is either accepted or rejected. 


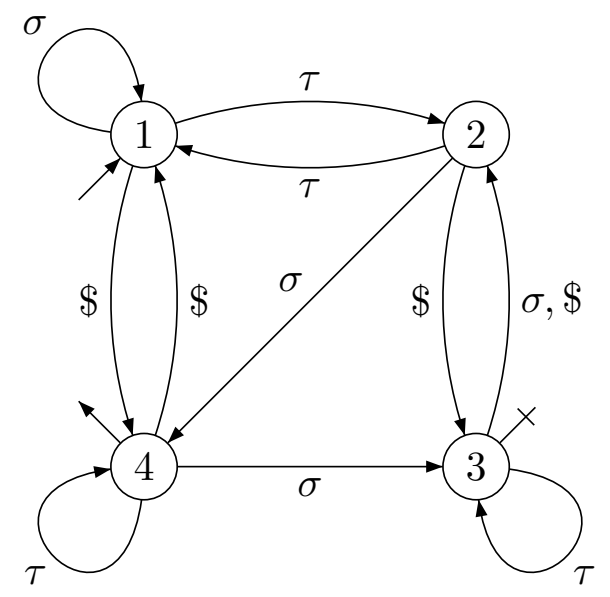

Figure 1: A reversible finite automaton.

In the example of Figure 1, state 4 is accepting and state 3 is rejecting. States 1 and 2 are non-halting.

A reversible finite automaton is called end-decisive [9], if it accepts a word only after reading the end-marker \$. Dually, if the automaton rejects a word only after reading \$, it is called co-end-decisive. If a reversible finite automaton is either end-decisive or co-end-decisive, it will be called a deterministic Brodsky-Pippenger automaton (DBPA).

It can be noticed that any RFA $\mathcal{A}=\left(Q, \Sigma \cup\{\$\}, q_{0}, Q_{a}, Q_{r}, \cdot\right)$ can be transformed into a classical finite automaton $\mathcal{B}=\left(Q, \Sigma, q_{0}, F,{ }_{\mathcal{B}}\right)$, where $F=Q_{a} \cup\left\{q \in Q_{n} \mid q \cdot \$ \in Q_{a}\right\}$ and the new transition function is defined in the following way: for all $\sigma \in \Sigma$ and $q \in Q$,

$$
q \cdot \cdot_{\mathcal{B}} \sigma= \begin{cases}q \cdot \sigma & \text { if } q \text { is non-halting } \\ q & \text { if } q \text { is halting. }\end{cases}
$$

By eliminating in $\mathcal{B}$ the states which are not accessible from the initial state, we obtain an automaton $\mathcal{A}^{\prime}=\left(Q^{\prime}, \Sigma, q_{0}, F^{\prime}, \cdot\right)$, where $F^{\prime}=Q^{\prime} \cap F$, which recognizes the same language as $\mathcal{A}$. For instance, if $\mathcal{A}$ is the automaton represented in Figure 1, the automata $\mathcal{B}$ and $\mathcal{A}^{\prime}$ are represented in Figure 2. 

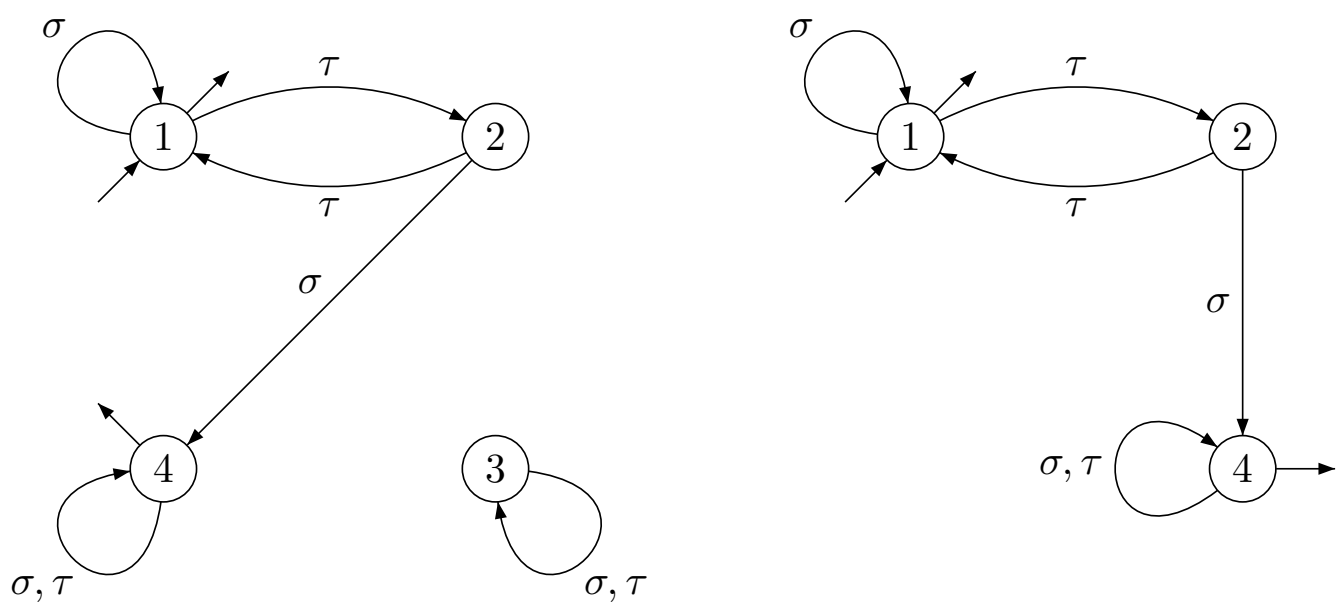

Figure 2: The automata $\mathcal{B}$ and $\mathcal{A}^{\prime}$.

Let us call such automaton $\mathcal{A}^{\prime}$ trivial if $\left|Q^{\prime}\right|=1$. A state $q$ such that, for every $\sigma \in \Sigma, q \cdot{ }_{\mathcal{B}} \sigma=q$, will be called absorbing.

Proposition 2.2. If $\mathcal{A}^{\prime}$ is non-trivial, a state of $Q^{\prime}$ is absorbing if and only if it is halting.

Proof. By the above construction, any halting state of $Q^{\prime}$ is absorbing.

Let $q$ be a state of $Q^{\prime}$. Since $q$ is accessible from $q_{0}\left(\right.$ in $\left.\mathcal{A}^{\prime}\right)$, there exists a word of minimal length $u=\sigma_{1} \cdots \sigma_{k} \in \Sigma^{*}$ such that $q_{0}{ }^{\cdot \mathcal{B}} u=q$. Then, for $0 \leqslant i \leqslant k-1$, the states $q_{0}{ }^{\cdot \mathcal{B}} \sigma_{1} \cdots \sigma_{i}$ are non-halting, for otherwise $q_{0} \cdot \mathcal{B} \sigma_{1} \cdots \sigma_{i} \sigma_{i+1}=\left(q_{0} \cdot_{\mathcal{B}} \sigma_{1} \cdots \sigma_{i}\right) \cdot \sigma_{i+1}=q_{0} \cdot_{\mathcal{B}} \sigma_{1} \cdots \sigma_{i}$, a contradiction, since $u$ is of minimal length. It follows that $q_{0} \cdot \mathcal{B} u=q_{0} \cdot u=q$.

If now $q$ is absorbing and non-halting, it follows from (2.3) that for $1 \leqslant i \leqslant k, q \cdot{ }_{\mathcal{B}} \sigma_{i}=q=q \cdot \sigma_{i}$. Thus $q_{0} \cdot u=q \cdot u=q$, and Condition (2.1) implies that $q=q_{0}$. Therefore $q_{0}$ is absorbing and hence $\mathcal{A}^{\prime}$ is trivial, a contradiction.

Consider the non-absorbing states of $\mathcal{A}^{\prime}$, which are also, by Proposition 2.2, the non-halting states. It follows from (2.3) that each letter of $\Sigma$ acts on these states as a partial injective function. All the absorbing states in $F^{\prime}$ are equivalent, so they can be merged. The same applies to non-final absorbing states.

The resulting deterministic automaton is equivalent to $\mathcal{A}$. It has at most two absorbing states and each letter defines a partial injective function on the set of non-absorbing states. An automaton with these properties will be called a classical reversible finite automaton (CRFA). Thus we have established the first part of the following result:

Proposition 2.3. Any RFA is equivalent to some CRFA. Conversely, any CRFA is equivalent to some RFA.

Proof. To complete the proof of the proposition, it remains to show that any CRFA $\mathcal{C}$ can be transformed into an equivalent reversible finite automaton $A=\left(Q, \Sigma \cup\{\$\}, s_{0}, Q_{a}, Q_{r}, \cdot\right)$, constructed as follows. Initially let $Q$ be the set of non-absorbing states of $\mathcal{C}$, let $Q_{a}=Q_{r}=\emptyset$ and restrict the transition function of $\mathcal{C}$ to $Q$. For each transition $e=p \stackrel{\sigma}{\longrightarrow} q$ of $\mathcal{C}$ such that $p$ is non-absorbing and $q$ is absorbing, add a 
new state $r_{e}$ to $Q$ and create a new transition $p \cdot \sigma=r_{e}$. If $q$ is final in $\mathcal{C}$, add $r_{e}$ to $Q_{a}$, and to $Q_{r}$, otherwise. Further, for any non-absorbing state $p$ of $\mathcal{C}$, add a new state $q$ to $Q$ and create a transition $p \cdot \$=q$. If $p$ is final in $\mathcal{C}$, add $q$ to $Q_{a}$, and to $Q_{r}$, otherwise. Now the automaton $\mathcal{A}$ recognizes the same language as $\mathcal{C}$. Each letter of $\Sigma \cup\{\$\}$ still defines a partial injective function, which can now be completed to a bijection in an arbitrary way.

If a CRFA has no absorbing states, it is a group automaton (all letters define permutations on the set of states) and it recognizes a group language. If it has at most one absorbing state, it will be called an injective finite automaton (IFA), to suggest the connection of this model with partial injective functions. Similarly as RFA are equivalent to CRFA, IFA are equivalent to DBPA. We call IFA-A (resp. IFA-R) an injective automaton whose absorbing state (if it exists) is final (resp. nonfinal). IFA-A are equivalent to co-end-decisive automata and IFA-R to end-decisive automata.
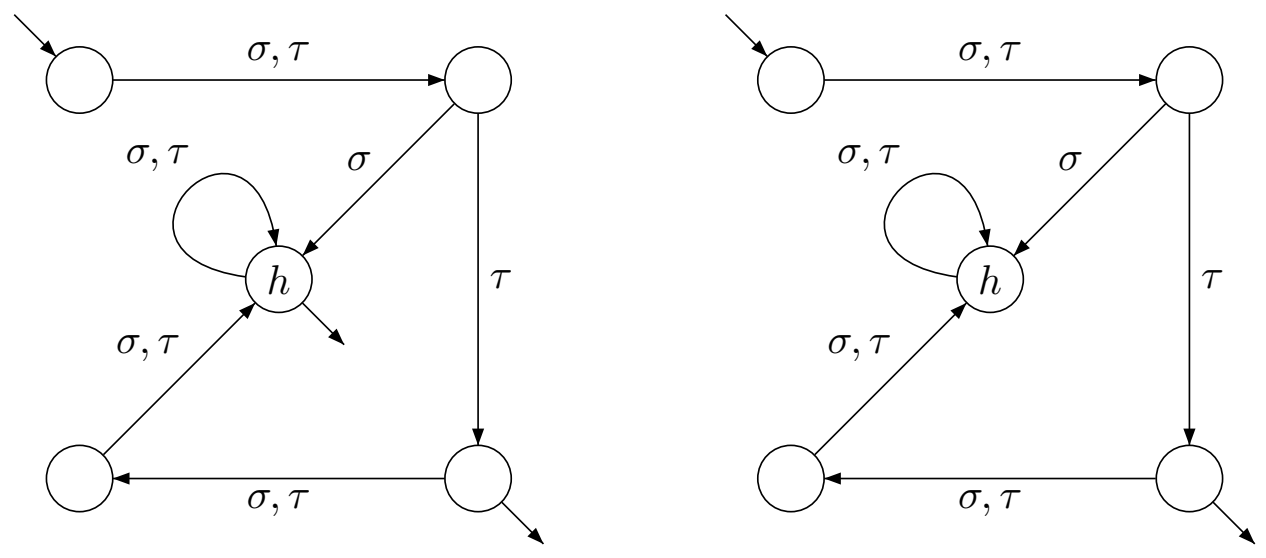

Figure 3: An IFA-A (on the left) and an IFA-R (on the right).

\section{Varieties}

If $x$ is an element of a monoid $M$, we denote by $x^{\omega}$ the unique idempotent of the subsemigroup of $M$ generated by $x$.

An ordered monoid $(M, \leqslant)$ is a monoid $M$ equipped with a stable order relation $\leqslant$ on $M$ which means that, for all $u, v, x \in M, u \leqslant v$ implies $u x \leqslant v x$ and $x u \leqslant x v$.

Let $M$ be a monoid and let $s$ be an element of $M$. An inverse of $s$ is an element $\bar{s}$ such that $s \bar{s} s=s$ and $\bar{s} s \bar{s}=\bar{s}$. An inverse monoid is a monoid in which every element has exactly one inverse. It is well known that the relation $\leqslant$ defined on an inverse monoid $M$ by

$$
x \leqslant y \text { if and only if } x=y e \text { for some idempotent } e \text { of } M
$$

is a stable partial order, called the natural order of $M$.

Following [22], we call ordered inverse monoid an inverse monoid $M$, equipped with its natural order. We also call dually ordered inverse monoid an inverse monoid ordered by the dual order of its natural order. 
A general overview on varieties of finite semigroups and monoids is given in [17], whereas introduction to varieties of ordered semigroups and monoids can be found in [20].

Given a monoid $M$, one may consider it to be an ordered monoid $(M,=)$ with equality as order relation. Given a variety of monoids $\mathbf{V}$, one may use the same notation for the variety of ordered monoids generated by $(M,=)$, where $M \in \mathbf{V}$. Both varieties satisfy the same identities (inequalities) and are related to the same class of regular languages.

Given two varieties of ordered monoids $\mathbf{V}$ and $\mathbf{W}$, their semidirect product $\mathbf{V} * \mathbf{W}$ is defined as in [22].

Theorem 3.1. The semidirect product is associative. Given varieties of ordered monoids $\mathbf{U}, \mathbf{V}$ and $\mathbf{W}$, $(\mathbf{U} * \mathbf{V}) * \mathbf{W}=\mathbf{U} *(\mathbf{V} * \mathbf{W})$.

Proof. An analogous statement for ordered semigroups is implied by the theorems in [22, Section 3]. The proofs of those results easily extend to the case of ordered monoids.

Definition 3.2. For any ordered monoid $M$, let $M^{\dagger}$ be the ordered monoid equipped with the dual order of $M$, that is, for any $x, y$ in $M, x \leqslant_{M^{\dagger}} y$ if and only if $y \leqslant_{M} x$.

Definition 3.3. For any variety of ordered monoids $\mathbf{V}$, let $\mathbf{V}^{\dagger}=\left\{M \mid M^{\dagger} \in \mathbf{V}\right\}$.

The following proposition is a direct consequence of the definitions above:

Proposition 3.4. (1) For any ordered monoid $M, M^{\dagger \dagger}=M$;

(2) For any variety of ordered monoids $\mathbf{V}, \mathbf{V}^{\dagger}$ is also a variety of ordered monoids and $\mathbf{V}^{\dagger \dagger}=\mathbf{V}$;

(3) For any varieties of ordered monoids $\mathbf{V}$ and $\mathbf{W},(\mathbf{V} * \mathbf{W})^{\dagger}=\mathbf{V}^{\dagger} * \mathbf{W}^{\dagger}$;

(4) For any variety of monoids $\mathbf{V}, \mathbf{V}^{\dagger}=\mathbf{V}$.

In this paper, we use the following varieties of ordered monoids, which are defined by some simple identities:

(1) $\mathbf{G}=\left[\left[x^{\omega}=1\right]\right]$, the variety of groups;

(2) $\mathbf{J}_{\mathbf{1}}=\left[\left[x^{2}=x, x y=y x\right]\right]$, the variety of commutative and idempotent monoids;

(3) $\mathbf{J}_{\mathbf{1}}^{+}=\left[\left[x^{2}=x, x \leqslant 1\right]\right]$, the variety of ordered idempotent monoids in which the identity is the maximum element. Order implies $x y \leqslant y, x y \leqslant x$, and since the monoids are idempotent, $x y \leqslant y x$. Hence $x y=y x$, and $\mathbf{J}_{\mathbf{1}}^{+} \subset \mathbf{J}_{\mathbf{1}}$;

(4) $\mathbf{J}_{\mathbf{1}}^{-}=\left[\left[x^{2}=x, 1 \leqslant x\right]\right]$, the variety of ordered idempotent monoids in which the identity is the minimum element. So $\mathbf{J}_{\mathbf{1}}^{-}=\left(\mathbf{J}_{\mathbf{1}}^{+}\right)^{\dagger}$. Similarly, $\mathbf{J}_{\mathbf{1}}^{-} \subset \mathbf{J}_{\mathbf{1}}$;

(5) $\mathbf{R}_{\mathbf{1}}=[[x y x=x y]]$, the variety of idempotent and $\mathcal{R}$-trivial monoids;

(6) ECom $=\left[\left[x^{\omega} y^{\omega}=y^{\omega} x^{\omega}\right]\right]$, the variety of monoids with commuting idempotents: the set of idempotents forms a submonoid which belongs to the variety $\mathbf{J}_{\mathbf{1}}$. This variety is known [7] to be equal to Inv, the variety of monoids generated by inverse monoids. Further, by [14], Inv $=\mathbf{J}_{\mathbf{1}} * \mathbf{G}=\mathbf{E C o m}$; 
(7) $\mathbf{E C o m}^{+}=\left[\left[x^{\omega} y^{\omega}=y^{\omega} x^{\omega}, x^{\omega} \leqslant 1\right]\right]$, the variety of ordered monoids whose idempotents form an ordered submonoid which belongs to the variety $\mathbf{J}_{1}^{+}$. This variety is known [22] to be equal to $\mathbf{I n v}^{+}$, the variety of ordered monoids generated by ordered inverse monoids. Moreover, $\mathbf{I n v}^{+}=$ $\mathbf{J}_{1}^{+} * \mathbf{G}=\mathbf{E C o m}^{+}$;

(8) $\mathbf{E C o m}^{-}=\left[\left[x^{\omega} y^{\omega}=y^{\omega} x^{\omega}, 1 \leqslant x^{\omega}\right]\right]$, the variety of ordered monoids whose idempotents form an ordered submonoid which belongs to the variety $\mathbf{J}_{\mathbf{1}}^{-}$. So $\mathbf{E C o m}^{-}=\left(\mathbf{E C o m}^{+}\right)^{\dagger}$. By Proposition 3.4, this variety is equal to $\mathbf{I n v} \mathbf{v}^{-}=\left(\mathbf{I n v}^{+}\right)^{\dagger}$, the variety of ordered monoids generated by dually ordered inverse monoids. Moreover, $\mathbf{I n v}^{-}=\mathbf{J}_{\mathbf{1}}^{-} * \mathbf{G}=\mathbf{E C o m}^{-}$;

(9) $\mathbf{E R}_{\mathbf{1}}=\left[\left[x^{\omega} y^{\omega} x^{\omega}=x^{\omega} y^{\omega}\right]\right]$, the variety of monoids whose idempotents form a submonoid which belongs to the variety $\mathbf{R}_{\mathbf{1}}$. Results in [2,1] imply that this variety is equal to $\mathbf{R}_{\mathbf{1}} * \mathbf{G}$;

(10) $\left.\mathbf{J}=\left[\left[x^{\omega} x=x^{\omega},(x y)^{\omega}=(y x)^{\omega}\right]\right]=\left[(x y)^{\omega} x=(x y)^{\omega}, x(y x)^{\omega}=(y x)^{\omega}\right]\right]$, the variety of $\mathcal{J}$-trivial monoids [10];

(11) $\mathbf{J}^{+}=[[x \leqslant 1]$, the variety of ordered monoids in which the identity is the maximum element. As noted in [22], $\mathbf{J}^{+} \subset \mathbf{J}$;

(12) $\mathbf{J}^{-}=[[1 \leqslant x]$, the variety of ordered monoids in which the identity is the minimum element. Similarly, $\mathbf{J}^{-}=\left(\mathbf{J}^{+}\right)^{\dagger}$ and $\mathbf{J}^{-} \subset \mathbf{J}$;

(13) $\mathbf{R}=\left[\left[(x y)^{\omega} x=(x y)^{\omega}\right]\right]$, the variety of $\mathcal{R}$-trivial monoids.

In the next section, we elaborate upon the results which in turn depend on the Vagner-Preston theorem $[26,25]$. By the Vagner-Preston theorem, monoids in $\mathbf{I n v}, \mathbf{I n v}{ }^{+}, \mathbf{I n v} \mathbf{v}^{-}$are generated by transition monoids of IFA, IFA-A, IFA-R, respectively.

\section{Injective Finite Automata}

In this section we shall describe the languages recognized by IFA, as well as an algebraic characterization of the Boolean closure of this class of languages. The transition monoid generated by an injective automaton is isomorphic to a submonoid of the monoid of injective partial functions from a finite set into itself, which justifies the name chosen for the model.

The classes of languages recognized by IFA-A and IFA-R will be denoted by $\mathbf{L}$ and $\mathbf{L}^{\mathbf{c}}$, respectively. The intersection of $\mathbf{L}$ and $\mathbf{L}^{\mathbf{c}}$ is the class of group languages. Recall that a class of languages is closed under inverse morphism if for any monoid morphism $\varphi: \Sigma^{*} \rightarrow \Gamma^{*}$ and for any language $L$ in the class, the language $\varphi^{-1}(L)$ is also in the class. Given a word $u$ and a language $L$ of $\Sigma^{*}$, recall that the quotient of $L$ by $u$ on the left (resp. right) is the language $u^{-1} L=\left\{v \in \Sigma^{*} \mid u v \in L\right\}$ (resp. $L u^{-1}=\left\{v \in \Sigma^{*} \mid v u \in L\right\}$ ).

Theorem 4.1. The classes $\mathbf{L}$ and $\mathbf{L}^{\mathbf{c}}$ are closed under inverse morphisms and word quotients. Furthermore, the class $\mathbf{L}$ is closed under finite union and the class $\mathbf{L}^{\mathbf{c}}$ under finite intersection.

Proof. Let $\varphi: \Sigma^{*} \rightarrow \Gamma^{*}$ be a monoid morphism and let $\mathcal{A}=(Q, \Gamma, i, F, \cdot)$ be an automaton recognizing a language $L$ of $\Gamma^{*}$. Then the language $\varphi^{-1}(L)$ is recognized by the automaton $\mathcal{B}=\left(Q, \Sigma, i, F,{ }_{\mathcal{B}}\right)$, where 
the transition function is given, for each $q \in Q$ and $\sigma \in \Sigma$, by $q \cdot{ }_{\mathcal{B}} \sigma=q \cdot \varphi(\sigma)$. It follows that if $\mathcal{A}$ is an IFA-A (resp. IFA-R), then so is $\mathcal{B}$. Therefore both classes $\mathbf{L}$ and $\mathbf{L}^{\mathbf{c}}$ are closed under inverse morphisms.

Let $\mathcal{A}=(Q, \Sigma, i, F, \cdot)$ be an automaton recognizing a language $L$ and let $u$ be a word. Then the language $u^{-1} L$ is recognized by the automaton $(Q, \Sigma, i \cdot u, F, \cdot)$ and the language $L u^{-1}$ is recognized by the automaton $\left(Q, \Sigma, i, F^{\prime}, \cdot\right)$, where $F^{\prime}=\{q \in Q \mid q \cdot u \in F\}$. It follows that the classes $\mathbf{L}$ and $\mathbf{L}^{\mathbf{c}}$ are closed under quotients.

Consider two languages $L_{1}$ and $L_{2}$, recognized by the deterministic automata $\mathcal{A}_{1}=\left(Q_{1}, \Sigma, i_{1}, F_{1}, \cdot\right)$ and $\mathcal{A}_{2}=\left(Q_{2}, \Sigma, i_{2}, F_{2}, \cdot\right)$, respectively.

First assume that $\mathcal{A}_{1}$ and $\mathcal{A}_{2}$ are IFA-A. The language $L_{1} \cup L_{2}$ is recognized by the automaton $\left(Q_{1} \times Q_{2}, \Sigma,\left(i_{1}, i_{2}\right),\left(Q_{1} \times F_{2}\right) \cup\left(F_{1} \times Q_{2}\right), \cdot\right)$, where the transition function is defined by $\left(q_{1}, q_{2}\right) \cdot \sigma=$ $\left(q_{1} \cdot \sigma, q_{2} \cdot \sigma\right)$. This automaton is not an IFA-A. However, all of its states having at least one absorbing component are equivalent and therefore can be merged to an absorbing final state. The resulting automaton is an IFA-A which recognizes $L_{1} \cup L_{2}$.

Suppose now that $\mathcal{A}_{1}$ and $\mathcal{A}_{2}$ are IFA-R. The language $L_{1} \cap L_{2}$ is recognized by the product automaton $\left(Q_{1} \times Q_{2}, \Sigma,\left(i_{1}, i_{2}\right), F_{1} \times F_{2}, \cdot\right)$. All of its states having at least one absorbing component are equivalent and therefore can be merged to an absorbing non-final state. The resulting automaton is an IFA-R which recognizes $L_{1} \cap L_{2}$.

Theorem 4.2. A language of $\Sigma^{*}$ is in $\mathbf{L}$ if and only if it is of the form $L_{0} \cup\left(\cup_{\sigma \in \Sigma} L_{\sigma} \sigma \Sigma^{*}\right)$, where $L_{0}$ and the $L_{\sigma}$ are group languages.

Proof. First, if $L \subset \Sigma^{*}$ is a group-language and $\sigma \in \Sigma$, the languages $L$ and $L \sigma \Sigma^{*}$ are recognized by IFA-A and therefore are in $\mathbf{L}$. Since by Theorem $4.1, \mathbf{L}$ is closed under finite union, the languages described in the statement are in $\mathbf{L}$.

Consider now a language $L$ recognized by an IFA-A $\mathcal{A}=\left(Q, \Sigma, q_{0}, F, \cdot\right)$ having an absorbing state $h$. Let $P=Q \backslash\{h\}$. Each letter of $\Sigma$ induces an injective partial map on $P$. Completing these partial maps to bijections in an arbitrary way, we obtain a bijective automaton $\mathcal{B}=\left(P, \Sigma, \cdot_{\mathcal{B}}\right)$. Let $L_{0}$ be the language recognized by the automaton $\mathcal{A}_{0}=\left(P, \Sigma, q_{0}, F \backslash\{h\},{ }_{\mathcal{B}}\right)$ and, for each letter $\sigma \in \Sigma$, let $L_{\sigma}$ be the language recognized by the automaton $\mathcal{A}_{\sigma}=\left(P, \Sigma, q_{0}, F_{\sigma},{ }^{\cdot \mathcal{B}}\right)$, where $F_{\sigma}=\{q \in P \mid q \cdot \sigma=h\}$. If $L$ is the language recognized by the IFA-A represented in Figure 3 , the three automata $\mathcal{A}_{0}, \mathcal{A}_{\sigma}$ and $\mathcal{A}_{\tau}$ are pictured in Figure 4. Then by construction, $L=L_{0} \cup \bigcup_{\sigma \in \Sigma^{*}} L_{\sigma} \sigma \Sigma^{*}$.

Corollary 4.3. A language of $\Sigma^{*}$ is recognized by an IFA-R if and only if it can be written as $L_{0} \cap$ $\left(\bigcap_{\sigma \in \Sigma}\left(L_{\sigma} \sigma \Sigma^{*}\right)^{c}\right)$, where $L_{0}$ and the $L_{\sigma}$ are group languages.

So the class of languages recognized by IFA is characterized by Theorem 4.2 and Corollary 4.3.

By Theorem 4.1, $\mathbf{L}$ ( $\mathbf{L}^{\mathbf{c}}$, respectively) is closed under finite union (finite intersection), inverse morphisms and word quotients. Nevertheless, $\mathbf{L}\left(\mathbf{L}^{\mathbf{c}}\right.$, respectively) does not form a disjunctive (conjunctive) variety in the sense of Polák [24], since it is not closed under inverse free semiring morphisms $\psi^{(-1)}\left(\psi^{[-1]}\right)$ as defined there. Using notations from [24], we consider a free semiring morphism $\psi: F\left(a^{*}\right) \longrightarrow F\left(\{a, b\}^{*}\right)$, where $\psi(\{a\})=\{a, b\}$. Consider the group languages recognized by the following minimal deterministic automaton: $Q=\left\{q_{0}, q_{1}, q_{2}, q_{3}\right\}, \Sigma=\{a, b\}, q_{0} \cdot a=q_{0} \cdot b=q_{1}, q_{1} \cdot a=q_{0}$, $q_{1} \cdot b=q_{2}, q_{2} \cdot a=q_{2} \cdot b=q_{3}, q_{3} \cdot a=q_{2}, q_{3} \cdot b=q_{0}$. The initial state is $q_{0}$. This automaton recognizes a 


\section{Varieties Generated by Certain Models of Reversible Finite Automata}
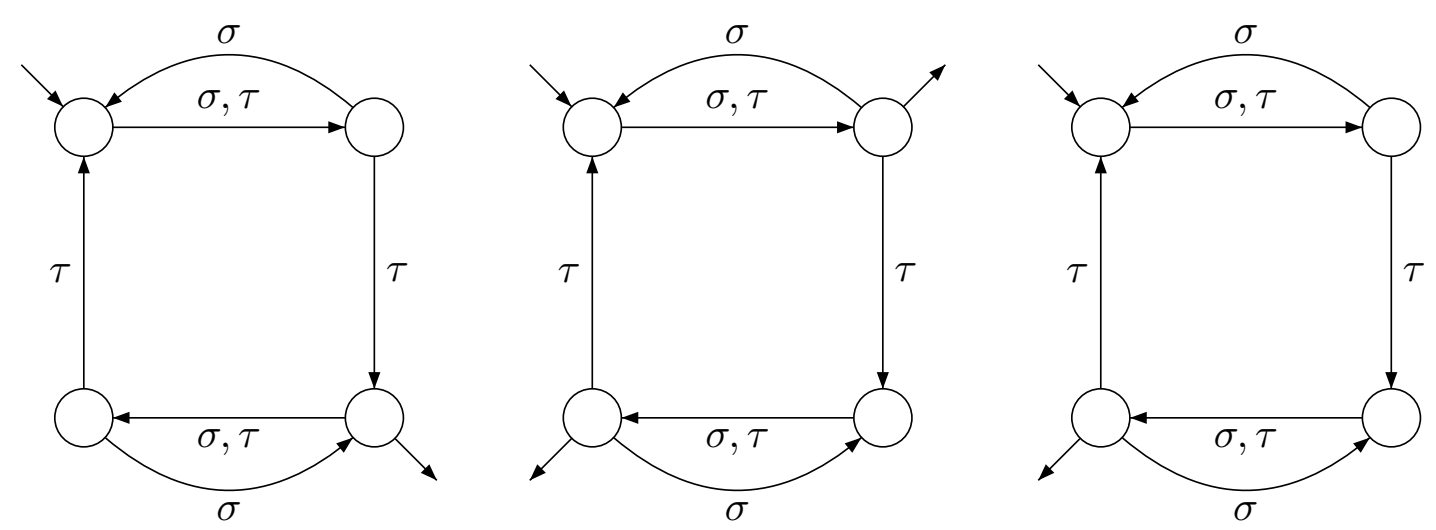

Figure 4: The automata $\mathcal{A}_{0}, \mathcal{A}_{\sigma}$ and $\mathcal{A}_{\tau}$, respectively.

group language $L_{1}$, if $F=\left\{q_{2}\right\}$, and a group language $L_{2}$, if $F=\left\{q_{0}, q_{1}, q_{3}\right\}$. Now $\psi^{(-1)}\left(L_{1}\right)=a a(a a)^{*}$ and $\psi^{[-1]}\left(L_{2}\right)=\{\varepsilon\} \cup a(a a)^{*}$. By Theorem 6.1, none of these two languages is recognized by an RFA. Hence $\mathbf{L}$ is not a disjunctive variety and $\mathbf{L}^{\mathbf{c}}$ is not a conjunctive variety (in the sense of Polák).

Consider the closure of $\mathbf{L}$ under finite intersection. The resulting class of languages is a positive variety of languages. By [23, Theorem 4.4], the corresponding variety of ordered monoids is $\mathbf{J}_{1}^{+} * \mathbf{G}=\mathbf{E} \mathbf{C o m}{ }^{+}$. Combining this result with the description of the languages of $\mathbf{L}$ given by Theorem 4.2, we obtain the following result:

Proposition 4.4. Let $Z$ be a language of $\Sigma^{*}$. The following conditions are equivalent:

(1) $Z$ belongs to the closure of $\mathbf{L}$ under finite intersection;

(2) $Z$ is a positive Boolean combination of languages of the form $L$ or $L \sigma \Sigma^{*}$, where $L$ is a group language;

(3) The syntactic ordered monoid of $Z$ belongs to the variety $\mathbf{E C o m}^{+}$.

Similarly, the closure of $\mathbf{L}^{\mathbf{c}}$ under finite union is exactly the class of languages recognized by Pin's reversible automata and the corresponding variety of ordered monoids is $\mathbf{E C o m}^{-}[18,19]$.

Finally, by [14], the closure of $\mathbf{L}$ or $\mathbf{L}^{\mathbf{c}}$ under Boolean operations corresponds to the monoid variety $\mathbf{J}_{1} * \mathbf{G}=\mathbf{E C o m}$.

\section{Reversible Finite Automata}

The class of languages recognized by CRFA (which, by Proposition 2.3, is also the class of languages recognized by RFA) will be denoted by $\mathbf{K}$.

This section gives a necessary condition for membership in $\mathbf{K}$, as well as an algebraic characterization of the Boolean closure $\overline{\mathbf{K}}$ of this class of languages. 
Theorem 5.1. Any language of $\Sigma^{*}$ recognized by a CRFA can be written as $K_{0} \cup K_{1} \sigma_{1} \Sigma^{*} \cup \ldots \cup K_{k} \sigma_{k} \Sigma^{*}$, where $K_{0}, \ldots, K_{k} \in \mathbf{L}^{\mathbf{c}}$ and $\sigma_{1}, \ldots, \sigma_{k}$ are letters.

Proof. Consider a language $Z$ recognized by a CRFA $\mathcal{A}=\left(Q, \Sigma, q_{0}, F, \cdot\right)$. If $\mathcal{A}$ has less than two absorbing states, the result follows from Theorem 4.2. Hence assume that $\mathcal{A}$ has two absorbing states: a non-final state $g$ and a final state $h$. Let $J=Q \backslash\{h\}$. We first decompose $Z$ as the union of two languages $K_{0}$ and $Z_{1}$. The language $K_{0}$ is recognized by the automaton $\mathcal{A}_{0}=\left(J, \Sigma, q_{0}, F \backslash\{h\}, .^{\prime}\right)$, where

$$
q \cdot^{\prime} \sigma= \begin{cases}q \cdot \sigma & \text { if } q \cdot \sigma \in J \\ g & \text { otherwise. }\end{cases}
$$

Then $\mathcal{A}_{0}$ is an IFA-R and thus $K \in \mathbf{L}^{\mathbf{c}}$. The language $Z_{1}$ is recognized by the automaton $\mathcal{A}_{1}=$ $\left(Q, \Sigma, q_{0},\{h\}, \cdot\right)$. For each transition in

$$
T=\{(q, \sigma) \in J \times \Sigma \mid q \cdot \sigma=h\}
$$

create an automaton $\mathcal{A}_{q, \sigma}=\left(Q, \Sigma, q_{0},\{h\},{ }_{q, \sigma}\right)$, where

$$
p \cdot_{q, \sigma} \tau= \begin{cases}p \cdot \tau & \text { if }(p, \tau) \notin T \text { or }(p, \tau)=(q, \sigma) \\ g & \text { otherwise. }\end{cases}
$$

Denoting by $Z_{(q, \sigma)}$ the language recognized by $\mathcal{A}_{(q, \sigma)}$, we obtain

$$
Z=\bigcup_{(q, \sigma) \in T} Z_{(q, \sigma)}
$$

Further, $Z_{(q, \sigma)}=K_{q, \sigma} \sigma \Sigma^{*}$, where $K_{q, \sigma}$ is the language in $\mathbf{L}^{\mathbf{c}}$ that is recognized by the automaton

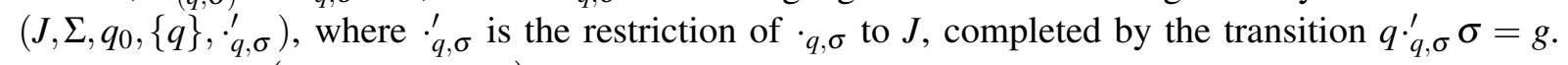
Hence $Z=K_{0} \cup\left(\underset{(q, \sigma) \in T}{\bigcup} K_{q, \sigma} \sigma \Sigma^{*}\right)$.

Note that given a language $K \subseteq \Sigma^{*}$ of $\mathbf{L}^{\mathbf{c}}$ and $\sigma \in \Sigma$, the language $K \sigma \Sigma^{*}$ is recognized by a CRFA.

Theorem 5.2. The class $\mathbf{K}$ is closed under complement, inverse of morphisms between free monoids and word quotients.

Proof. A multiplication of two transition matrices of a CRFA yields a transition matrix of a CRFA. Hence CRFA is closed under inverse free monoid morphisms. Closure under complement and word quotients is trivial.

Corollary 5.3. If a language of $\Sigma^{*}$ is recognized by a CRFA, then it can be written as $K_{0}^{c} \cap\left(K_{1} \sigma_{1} \Sigma^{*}\right)^{c} \cap$ $\cdots \cap\left(K_{k} \sigma_{k} \Sigma^{*}\right)^{c}$, where $k \geqslant 0, K_{0}, \ldots, K_{k} \in \mathbf{L}^{\mathbf{c}}$ and $\sigma_{1}, \ldots, \sigma_{k} \in \Sigma$.

Since $\mathbf{K}$ is closed under complement, its closure under positive Boolean operations (finite unions and intersections) is equal to its Boolean closure $\overline{\mathbf{K}}$.

Theorem 5.4. A language belongs to $\overline{\mathbf{K}}$ if and only if its syntactic ordered monoid belongs to $\mathbf{J}_{\mathbf{1}}^{+} *\left(\mathbf{J}_{\mathbf{1}}^{-} * \mathbf{G}\right)$. 
Proof. Let $L$ be a regular language and let $M(L)$ be its syntactic ordered monoid. If $L \in \overline{\mathbf{K}}$, then it is by Theorem 5.1 a positive Boolean combination of languages of the form $K$ or $K \sigma \Sigma^{*}$, where $K \in \mathbf{L}^{\mathbf{c}}$. Thus by [18, 19], $M(K) \in \mathbf{E} \mathbf{C o m}^{-}=\mathbf{J}_{\mathbf{1}}^{-} * \mathbf{G}$. Therefore by [23, Theorem 4.4], $M(L) \in \mathbf{J}_{\mathbf{1}}^{+} *\left(\mathbf{J}_{\mathbf{1}}^{-} * \mathbf{G}\right)$.

Suppose now that $M(L) \in \mathbf{J}_{\mathbf{1}}^{+} *\left(\mathbf{J}_{\mathbf{1}}^{-} * \mathbf{G}\right)$. Then by [23, Theorem 4.4], $L$ is a positive Boolean combination of languages of the form $Z$ or $Z \sigma \Sigma^{*}$, where $M(Z) \in \mathbf{J}_{\mathbf{1}}^{-} * \mathbf{G}$. Further, $Z$ is a positive Boolean combination of languages of the form $Y_{i}$ and $\left(Y_{j} \sigma \Sigma^{*}\right)^{c}$, where $Y_{i}, Y_{j}$ are group languages. So $Z=\bigcup_{i} K_{i}$, where $K_{i} \in \mathbf{L}^{\mathbf{c}}$. Now $Z \sigma \Sigma^{*}=\left(\bigcup_{i} K_{i}\right) \sigma \Sigma^{*}=\bigcup_{i}\left(K_{i} \sigma \Sigma^{*}\right)$. Hence $L \in \overline{\mathbf{K}}$.

By Theorem 3.1, $\mathbf{J}_{\mathbf{1}}^{+} *\left(\mathbf{J}_{\mathbf{1}}^{-} * \mathbf{G}\right)=\left(\mathbf{J}_{\mathbf{1}}^{+} * \mathbf{J}_{\mathbf{1}}^{-}\right) * \mathbf{G}$, hence it is of interest to describe the variety $\mathbf{J}_{\mathbf{1}}^{+} * \mathbf{J}_{\mathbf{1}}^{-}$. A first step is to prove the following lemma:

Lemma 5.5. Let $M$ be a monoid in $\mathbf{J}_{\mathbf{1}}^{+}$(whose operation is denoted additively) and let $N$ be a monoid in $\mathbf{J}_{\mathbf{1}}^{-}$. Then, for any left action of $N$ on $M$, for all $m \in M$ and $n \in N, m+n \cdot m=m$.

Proof. Let $m \in M$ and $n \in N$. Since $M \in \mathbf{J}_{1}^{+}, m \leqslant 0$. Since the order relation is stable under the action, we obtain $n \cdot m \leqslant 0$ and consequently, $m+n \cdot m \leqslant m$. On the other hand, since $N \in \mathbf{J}_{\mathbf{1}}^{-}, 1 \leqslant n$. Hence $m \leqslant n \cdot m$ and $m \leqslant m+n \cdot m$. Now $m \leqslant m+n \cdot m \leqslant m$, therefore $m+n \cdot m=m$.

Theorem 5.6. The following equalities hold: $\mathbf{J}_{\mathbf{1}}^{+} * \mathbf{J}_{\mathbf{1}}^{-}=\mathbf{J}_{\mathbf{1}}^{-} * \mathbf{J}_{\mathbf{1}}^{+}=\mathbf{R}_{\mathbf{1}}$.

Proof. We first prove the equality $\mathbf{J}_{\mathbf{1}}^{+} * \mathbf{J}_{\mathbf{1}}^{-}=\mathbf{R}_{\mathbf{1}}$.

Let $M \in \mathbf{J}_{\mathbf{1}}^{+}$and $N \in \mathbf{J}_{\mathbf{1}}^{-}$. Both $M$ and $N$ satisfy the identities $x y=y x$ and $x^{2}=x$. Let us verify that $M * N$ satisfies the identity $x y x=x y$. Let $\left(m_{1}, n_{1}\right),\left(m_{2}, n_{2}\right) \in M * N$. Using Lemma 5.5, we obtain

$$
\begin{aligned}
\left(m_{1}, n_{1}\right)\left(m_{2}, n_{2}\right)\left(m_{1}, n_{1}\right) & =\left(m_{1}+n_{1} \cdot m_{2}+\left(n_{1} n_{2}\right) \cdot m_{1}, n_{1} n_{2} n_{1}\right) \\
& =\left(m_{1}+\left(n_{1} n_{2}\right) \cdot m_{1}+n_{1} \cdot m_{2}, n_{1} n_{2}\right) \\
& =\left(m_{1}+n_{1} \cdot m_{2}, n_{1} n_{2}\right)=\left(m_{1}, n_{1}\right)\left(m_{2}, n_{2}\right)
\end{aligned}
$$

Thus $M * N \in \mathbf{R}_{\mathbf{1}}$. Since the variety $\mathbf{J}_{\mathbf{1}}^{+} * \mathbf{J}_{\mathbf{1}}^{-}$is generated by such semidirect products, it follows that $\mathbf{J}_{\mathbf{1}}^{+} * \mathbf{J}_{\mathbf{1}}^{-} \subseteq \mathbf{R}_{\mathbf{1}}$.

Let $U_{2}^{r}=\{1, a, b\}$ be the idempotent monoid defined by $a b=a$ and $b a=b$. It is well known (see [21], for instance) that the variety $\mathbf{R}_{\mathbf{1}}$ is generated by $U_{2}^{r}$. Proving that $\left(U_{2}^{r},=\right) \in \mathbf{J}_{\mathbf{1}}^{+} * \mathbf{J}_{\mathbf{1}}^{-}$will thus suffice to establish the inclusion $\mathbf{R}_{\mathbf{1}} \subseteq \mathbf{J}_{\mathbf{1}}^{+} * \mathbf{J}_{\mathbf{1}}^{-}$.

Let $U_{1}^{+}=(\{0, a\},+, \leqslant)$ (respectively $\left.U_{1}^{-}=(\{1, b\}, \leqslant)\right)$ be the idempotent ordered monoid whose operation is denoted additively (respectively multiplicatively) and order is defined by $a \leqslant 0$ (respectively $1 \leqslant b$ ). Thus $U_{1}^{+} \in \mathbf{J}_{1}^{+}$and $U_{1}^{-} \in \mathbf{J}_{\mathbf{1}}^{-}$. Define a left action of $U_{1}^{-}$on $U_{1}^{+}$by setting $1 \cdot 0=0, b \cdot 0=0$, $1 \cdot a=a, b \cdot a=0$. One can easily verify that the left action satisfies the conditions (1)-(6), defined for left actions of ordered monoids in [22]. Thus we have defined a semidirect product $U_{1}^{+} * U_{1}^{-}$. Let $1=(0,1), x=(0, b), y=(a, 1), z=(a, b)$ and consider the submonoid $T=\{1, x, z\}$ of $U_{1}^{+} * U_{1}^{-}$. Then the submonoid $M=\{(1,1),(x, z),(z, x)\}$ of $T \times T$ is isomorphic to the monoid $\left(U_{2}^{r},=\right)$. It follows that $\left(U_{2}^{r},=\right) \in \mathbf{J}_{\mathbf{1}}^{+} * \mathbf{J}_{\mathbf{1}}^{-}$and thus $\mathbf{R}_{\mathbf{1}} \subseteq \mathbf{J}_{\mathbf{1}}^{+} * \mathbf{J}_{\mathbf{1}}^{-}$.

Now $\mathbf{R}_{\mathbf{1}}=\mathbf{J}_{\mathbf{1}}^{+} * \mathbf{J}_{\mathbf{1}}^{-}$and hence by Proposition 3.4, $\mathbf{R}_{\mathbf{1}}=\mathbf{J}_{\mathbf{1}}^{-} * \mathbf{J}_{\mathbf{1}}^{+}$. 
The variety of monoids $\mathbf{R}_{\mathbf{1}}$ is defined by the identity $x y x=x y$. Hence by [2, Corollary 4.3] and [1, Exercise 10.2.4], $\mathbf{R}_{\mathbf{1}} * \mathbf{G}=\left[\left[x^{\omega} y^{\omega} x^{\omega}=x^{\omega} y^{\omega}\right]\right]=\mathbf{E R}_{\mathbf{1}}$.

This yields the following theorem, which essentially says that the languages recognized by RFA generate the variety $\mathbf{E R}_{\mathbf{1}}$ :

Theorem 5.7. A language is in $\overline{\mathbf{K}}$ if and only if its syntactic monoid belongs to the variety $\mathbf{E} \mathbf{R}_{\mathbf{1}}$.

\section{Algebraic Conditions}

Let us note that Ambainis and Freivalds have proved [5, Theorems 2 and 3] the following characterization for the class of languages recognized by RFA:

Theorem 6.1. Let $\mathcal{A}$ be the minimal automaton of a regular language $L$. Then $L$ is recognized by a reversible finite automaton if and only if for any states $q_{1}, q_{2}, q_{3}$ of $\mathcal{A}$, such that $q_{1} \neq q_{2}, q_{2} \neq q_{3}$, and for any input words $x, y, \mathcal{A}$ does not contain the following configuration: $q_{1} \cdot x=q_{2}, q_{2} \cdot x=q_{2}, q_{2} \cdot y=q_{3}$.

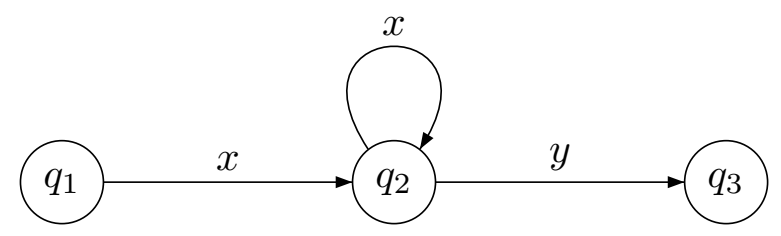

Figure 5: The forbidden configuration in a RFA.

The Ambainis-Freivalds condition can be translated into an algebraic condition. Let $L$ a regular language of $\Sigma^{*}$. We denote by $M(L)$ its syntactic monoid, by $\varphi: \Sigma^{*} \longrightarrow M(L)$ its syntactic morphism and by $P=\varphi(L)$ the syntactic image of $L$. Let $\sim_{r}$ be the right congruence on $M(L)$ defined by $s \sim_{r} t$ if and only if, for all $u \in M(L), s u \in P$ is equivalent to $t u \in P$.

Corollary 6.2. A language $L$ is recognized by a reversible finite automaton if and only if for all $s, t, u \in$ $M(L)$,

$$
s t^{\omega} \sim_{r} s \text { or } s t^{\omega} u \sim_{r} s t^{\omega} .
$$

Proof. Consider the minimal automaton $\left(Q, \Sigma, q_{0}, F, \cdot\right)$ of a language $L$. Due to Ambainis-Freivalds condition, a language is recognized by a reversible finite automaton if and only if for all $q_{1}, q_{2}, q_{3} \in Q$ and $x, y \in \Sigma^{*}$,

$$
q_{1} \cdot x=q_{2}, q_{2} \cdot x=q_{2} \text { and } q_{2} \cdot y=q_{3} \text { imply } q_{1}=q_{2} \text { or } q_{2}=q_{3}
$$

or, equivalently, for all $q \in Q$, for all $x, y \in \Sigma^{*}$,

$$
q \cdot x=q \cdot x^{2} \text { implies } q=q \cdot x \text { or } q \cdot x=q \cdot x y
$$

Now, choose $v \in \Sigma^{*}$ such that $q=q_{0} \cdot v$ and let $s=\varphi(v)$ and $t=\varphi(x)$. We claim that the condition $q \cdot x=q \cdot x^{2}$ is equivalent to $s t \sim_{r} s t^{2}$. Indeed, by the definition of the Nerode equivalence, the first 
condition means that, for every $y \in \Sigma^{*}, q_{0} \cdot v x y \in F$ if and only if $q_{0} \cdot v x^{2} y \in F$, or, equivalently, for all $u \in M(L)$, stu $\in P$ if and only if $s t^{2} u \in P$.

Therefore, Formula (6.3) can be rewritten as follows: for all $s, t, u \in M(L)$,

$$
s t \sim_{r} s t^{2} \text { implies } s \sim_{r} s t \text { or } s t \sim_{r} s t u
$$

which is in turn equivalent to: for all $s, t, u \in M(L)$,

$$
s \sim_{r} s t^{\omega} \text { or } s t^{\omega} \sim_{r} s t^{\omega} u
$$

Consider an injective automaton $\mathcal{A}$, which is not a group automaton, i.e., has one absorbing state. We assume that $\mathcal{A}$ is accessible. Then for any state $q$ and any word $w$, there exists $k>0$ such that $q \cdot w^{k}=q$ or $q \cdot w^{k}=h$, where $h$ is the absorbing state. Therefore we deduce that the absorbing state is accessible from any state. So the transition monoid $M(\mathcal{A})$ has a zero element ([17, Exercise 2.7]). Since $M(L)$ divides $M(\mathcal{A}), M(L)$ also has a zero element. One can view the syntactic monoid $M(L)$ as an automaton $(M(L), \Sigma, 1, P, \cdot)$, which recognizes $L$. Any of its states is accessible from the initial state 1 . The right equivalence class containing 0 corresponds to the absorbing state in the minimal automaton of $L$. All the absorbing states of $M(L)$ are in this class. Hence if for every $u, s t^{\omega} \sim_{r} s t^{\omega} u$, then $s t^{\omega} \sim_{r} 0$. Thus in the case of DBPA, Corollary 6.2 may be rewritten as follows:

Corollary 6.3. A language $L$ is recognized by a deterministic Brodsky-Pippenger automaton if and only if, for all $s, t \in M(L), s t^{\omega} \sim_{r} s$ or $s t^{\omega} \sim_{r} 0$.

If $L$ is a group language, $M(L)$ does not have a zero, so this condition reduces to: for all $s, t \in M(L)$, $s t^{\omega} \sim_{r} s$, which is in turn equivalent to $t^{\omega}=1$.

Finally, let us note one can prove the following theorem:

Theorem 6.4. Let $\mathcal{A}$ be the minimal automaton of a regular language $L$. Then $M(L) \in \mathbf{E R}_{\mathbf{1}}$ if and only if for all states $q_{1}, q_{2}, q_{3}$ of $\mathcal{A}$, such that $q_{2} \neq q_{3}$, and for all input words $x, y, \mathcal{A}$ does not contain the following configuration: $q_{1} \cdot y=q_{1}, q_{1} \cdot x=q_{2}, q_{2} \cdot x=q_{2}, q_{2} \cdot y=q_{3}, q_{3} \cdot y=q_{3}$.

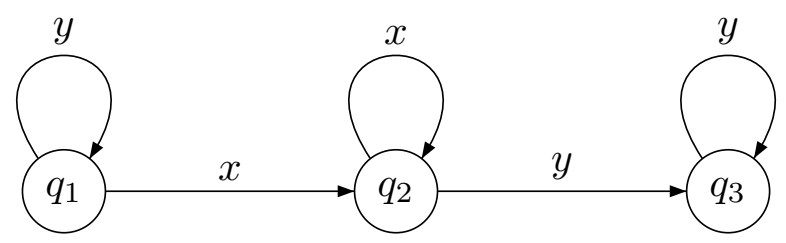

Figure 6: The forbidden configuration corresponding to $\mathbf{E R}_{\mathbf{1}}$.

One may notice similarities in Figures 5 and 6. 


\section{Semidirect Products of Ordered $\mathcal{J}$-Trivial Monoids of Opposite Order}

We proved in Section 5 that $\mathbf{J}_{\mathbf{1}}^{+} * \mathbf{J}_{\mathbf{1}}^{-}=\mathbf{J}_{\mathbf{1}}^{-} * \mathbf{J}_{\mathbf{1}}^{+}=\mathbf{R}_{\mathbf{1}}$. So it is reasonable to ask whether a similar equality holds for varieties of ordered $\mathcal{J}$-trivial monoids. Indeed, in this section we prove that $\mathbf{J}^{+} * \mathbf{J}^{-}=\mathbf{J}^{-} * \mathbf{J}^{+}=$ R.

Let us say that a regular language is $\mathcal{R}$-trivial (respectively $\mathcal{J}$-trivial) if it is recognized by an $\mathcal{R}$-trivial (respectively $\mathcal{J}$-trivial) monoid. We first recall some known characterizations of these languages (see $[10,17])$.

Theorem 7.1. A language of $A^{*}$ is $\mathcal{R}$-trivial if and only if it is a disjoint union of languages of the form $A_{0}^{*} a_{1} A_{1}^{*} a_{2} \cdots a_{n} A_{n}^{*}$ with $n \geqslant 0, a_{1}, \ldots, a_{n} \in A, A_{i} \subseteq A \backslash\left\{a_{i+1}\right\}$ for $0 \leqslant i \leqslant n-1$ and $A_{n} \subseteq A$.

Theorem 7.2 (Simon). A language of $A^{*}$ is $\mathcal{J}$-trivial if and only if it is a Boolean combination of languages of the form $A^{*} a_{1} A^{*} a_{2} \cdots a_{n} A^{*}$ with $n \geqslant 0$ and $a_{1}, \ldots, a_{n} \in A$.

We need a slightly different characterization of the $\mathcal{R}$-trivial languages, which requires a new definition.

Definition 7.3. An $\mathcal{R}$-basic language of $A^{*}$ is a language of the form

$$
A_{0}^{*} a_{1} A_{1}^{*} a_{2} \cdots A_{n-1}^{*} a_{n} A_{n}^{*} a_{n+1} A^{*}
$$

with $n \geqslant 0, a_{1}, \ldots, a_{n+1} \in A, A_{i}=A \backslash\left\{a_{i+1}\right\}$ for $0 \leqslant i \leqslant n-1$ and $A_{n} \subseteq A \backslash\left\{a_{n+1}\right\}$.

We are now ready to prove the announced description of $\mathcal{R}$-trivial languages.

Theorem 7.4. A language of $A^{*}$ is $\mathcal{R}$-trivial if and only if it is a positive Boolean combination of $\mathcal{R}$-basic and J-trivial languages.

Proof. Clearly, any $\mathcal{R}$-basic or $\mathcal{J}$-trivial language is $\mathcal{R}$-trivial. Taking into account Theorem 7.1, it suffices to show that the language

$$
L=A_{0}^{*} a_{1} A_{1}^{*} a_{2} \cdots a_{n} A_{n}^{*}
$$

with $n \geqslant 0, a_{1}, \ldots, a_{n} \in A, A_{i} \subseteq A \backslash\left\{a_{i+1}\right\}$ for $0 \leqslant i \leqslant n-1$ and $A_{n} \subseteq A$, is a finite intersection of $\mathcal{R}$-basic and $\mathcal{J}$-trivial languages. The minimal automaton $\mathfrak{A}$ of $L$ is represented in Figure 7, where $A_{i}^{\prime}=A \backslash\left(A_{i} \cup\left\{a_{i+1}\right\}\right)$ for $0 \leqslant i \leqslant n-1$ and $A_{n}^{\prime}=A \backslash A_{n}$. 


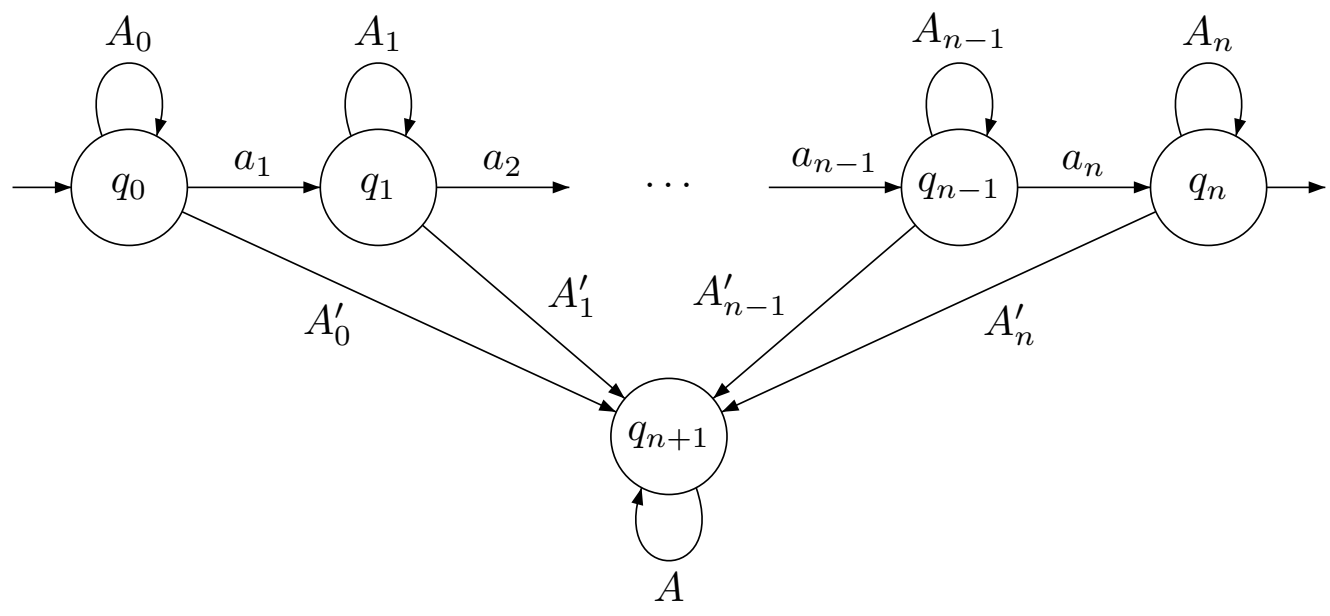

Figure 7: The automaton $\mathfrak{A}$.

We claim that

$$
L=\bigcap_{0 \leqslant i \leqslant n} L_{i}
$$

where, for $0 \leqslant i \leqslant n-1$,

$$
L_{i}=B_{0}^{*} a_{1} B_{1}^{*} a_{2} \cdots B_{i-1}^{*} a_{i} A_{i}^{*} a_{i+1} A^{*}
$$

and

$$
L_{n}=\left(B_{0}^{*} a_{1} B_{1}^{*} a_{2} \cdots B_{n-1}^{*} a_{n} A_{n}^{*} B_{n} A^{*}\right)^{c},
$$

with $B_{i}=A \backslash\left\{a_{i+1}\right\}$ for $0 \leqslant i \leqslant n-1$ and $B_{n}=A \backslash A_{n}$.

The languages $L_{0}, \ldots, L_{n-1}$ are $\mathcal{R}$-basic. Further, $L_{n}$ is $\mathcal{J}$-trivial since

$$
B_{0}^{*} a_{1} B_{1}^{*} a_{2} \cdots B_{n-1}^{*} a_{n} A_{n}^{*} B_{n} A^{*}=\bigcup_{a \in B_{n}} A^{*} a_{1} A^{*} a_{2} \cdots A^{*} a_{n} A^{*} a A^{*}
$$

The minimal automata $\mathfrak{A}_{i}$ of the languages $L_{i}$ are depicted in Figures 8 and 9. [ht]

Let $u \in L$. Taking $u$ as an input, the automaton $\mathfrak{A}$ halts in the final state $q_{n}$. Since for all $0 \leqslant i \leqslant n-1$, $A_{i} \subseteq B_{i}$, on the same input $u$, the automaton $\mathfrak{A}_{i}$ halts in $q_{i+1}$, which for $\mathfrak{A}_{i}$ is the final state. As for the automaton $\mathfrak{A}_{n}$, it halts in $q_{n}$ and thus accepts $u$. Therefore $u \in \bigcap_{0 \leqslant i \leqslant n} L_{i}$.

On the other hand, if $u \notin L$, then $\mathfrak{A}$ rejects $u$ and the computation halts in one of the states $q_{0}, \ldots, q_{n-1}$ or in $q_{n+1}$. If it halts in one of the states $q_{i}$, with $0 \leqslant i<n$, then the automaton $\mathfrak{A}_{i}$ also halts in $q_{i}$ and 
Marats Golovkins AND JEAN-ERIC Pin

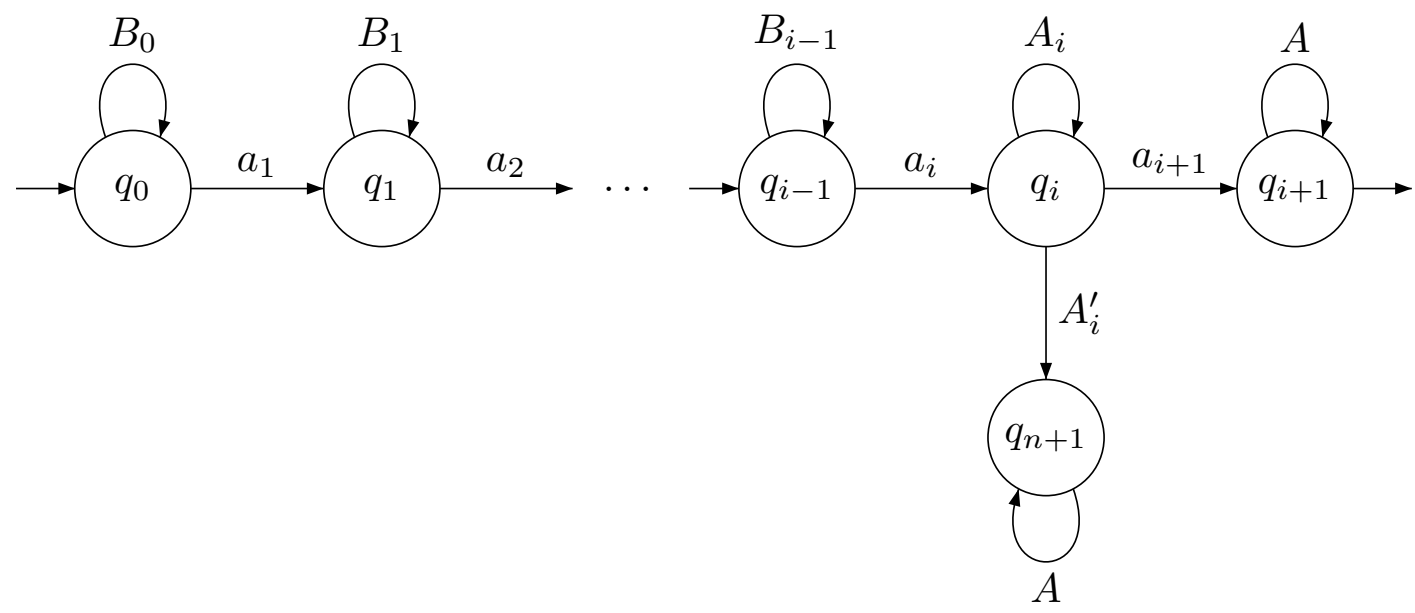

Figure 8: The automaton $\mathfrak{A}_{i}$, for $0 \leqslant i \leqslant n-1$.

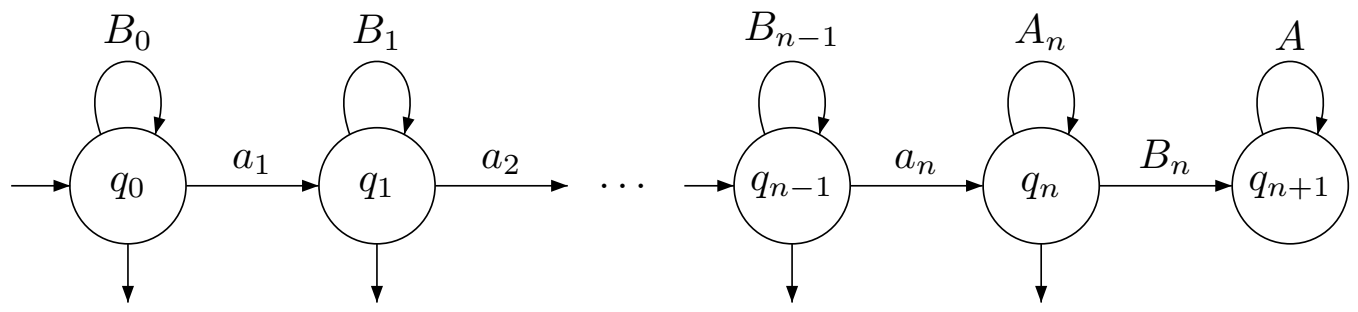

Figure 9: The automaton $\mathfrak{A}_{n}$.

rejects $u$. If it halts in $q_{n+1}$, then the path of label $u$ visits all the states of some sequence $q_{0}, \ldots, q_{i}$ before reaching $q_{n+1}$. In this case, the automaton $\mathfrak{A}_{i}$ visits the same sequence of states and hence rejects $u$. Altogether, there exists $i$ such that $u \notin L_{i}$ and so $u \notin \bigcap_{0 \leqslant i \leqslant n} L_{i}$. Therefore $L=\bigcap_{0 \leqslant i \leqslant n} L_{i}$.

We are now ready to prove the main result of this section.

Theorem 7.5. The following equalities hold: $\mathbf{J}_{\mathbf{1}}^{+} * \mathbf{J}=\mathbf{J}_{\mathbf{1}}^{-} * \mathbf{J}=\mathbf{J}^{+} * \mathbf{J}^{-}=\mathbf{J}^{-} * \mathbf{J}^{+}=\mathbf{R}$.

Proof. We first prove the equalities $\mathbf{J}_{\mathbf{1}}^{+} * \mathbf{J}=\mathbf{J}^{+} * \mathbf{J}^{-}=\mathbf{R}$.

It is proved in [10] that $\mathbf{R} * \mathbf{R}=\mathbf{R}$. Since $\mathbf{J} \subset \mathbf{R}, \mathbf{J}^{+} * \mathbf{J}^{-} \subseteq \mathbf{R}$ and $\mathbf{J}_{1}^{+} * \mathbf{J} \subseteq \mathbf{R}$. It remains to establish inclusions $\mathbf{R} \subseteq \mathbf{J}_{1}^{+} * \mathbf{J} \subseteq \mathbf{J}^{+} * \mathbf{J}^{-}$. As noted in [22], $\mathbf{J}=\mathbf{J}^{+} \vee \mathbf{J}^{-}$, whence $\mathbf{J} \subseteq \mathbf{J}^{+} * \mathbf{J}^{-}$. It follows from $\left[22\right.$, Corollary 4.3] that $\mathbf{J}_{\mathbf{1}}^{+} * \mathbf{J}^{+}=\mathbf{J}^{+}$. Thus $\mathbf{J}_{\mathbf{1}}^{+} * \mathbf{J} \subseteq \mathbf{J}_{\mathbf{1}}^{+} *\left(\mathbf{J}^{+} * \mathbf{J}^{-}\right)=\left(\mathbf{J}_{\mathbf{1}}^{+} * \mathbf{J}^{+}\right) * \mathbf{J}^{-}=\mathbf{J}^{+} * \mathbf{J}^{-}$.

Eilenberg's theorem and the results of [20] show that, to establish the inclusion $\mathbf{R} \subseteq \mathbf{J}_{\mathbf{1}}^{+} * \mathbf{J}$, it is sufficient to prove that any $\mathcal{R}$-trivial language $L$ is recognized by an ordered monoid in $\mathbf{J}_{1}^{+} * \mathbf{J}$. By Theorem 7.4, $L$ is a positive Boolean combination of $\mathcal{R}$-basic and $\mathcal{J}$-trivial languages. Consequently, it only remains to prove that any $\mathcal{R}$-basic language is recognized by a monoid in $\mathbf{J}_{1}^{+} * \mathbf{J}$. 
Let $K$ be an $\mathcal{R}$-basic language of $A^{*}$, say

$$
K=A_{0}^{*} a_{0} A_{1}^{*} a_{1} \cdots A_{n-1}^{*} a_{n-1} A_{n}^{*} a_{n} A^{*}
$$

with $n \geqslant 0, a_{0}, \ldots, a_{n} \in A, A_{i}=A \backslash\left\{a_{i}\right\}$ for $0 \leqslant i \leqslant n-1$ and $A_{n} \subseteq A \backslash\left\{a_{n}\right\}$. Then the language $K^{\prime}=A_{0}^{*} a_{0} A_{1}^{*} a_{1} \ldots A_{n-1}^{*} a_{n-1} A_{n}^{*}$ is $\mathcal{J}$-trivial and $K=K^{\prime} a_{n} A^{*}$. It follows now from [23, Theorem 4.4] that $K$ is recognized by a monoid in $\mathbf{J}_{\mathbf{1}}^{+} * \mathbf{J}$. Therefore $\mathbf{R}=\mathbf{J}_{\mathbf{1}}^{+} * \mathbf{J}=\mathbf{J}^{+} * \mathbf{J}^{-}$and hence by Proposition 3.4, $\mathbf{R}=\mathbf{J}_{\mathbf{1}}^{-} * \mathbf{J}=\mathbf{J}^{-} * \mathbf{J}^{+}$.

\section{References}

[1] J. AlmeIDA: Finite Semigroups and Universal Algebra. World Scientific, Singapore, 1994. 7, 12

[2] J. Almeida, J.E. Pin, AND P. WeIL: Semigroups whose idempotents form a subsemigroup. Math. Proc. Camb. Phil. Soc., 111:241-253, 1992. 7, 12

[3] A. Ambainis, M. Beaudry, M. Golovkins, A. ,Kikusts, M. Mercer, And D. Thérien: Algebraic results on quantum automata. In STACS 2004, volume 2996 of Lecture Notes in Computer Science, pp. 93-104, 2004. 1

[4] A. Ambainis, R.F. Bonner, R. Freivalds, And A. Kikusts: Probabilities to accept languages by quantum finite automata. In COCOON 1999, volume 1627 of Lecture Notes in Computer Science, pp. 174-183, 1999. 1

[5] A. Ambainis And R. Freivalds: 1-way quantum finite automata: Strengths, weaknesses and generalizations. In Proc. 39th FOCS, pp. 332-341, 1998. 1, 12

[6] A. Ambainis, A. Nayak, A. TA-Shma, And U. Vazirani: Dense quantum coding and quantum finite automata. Journal of the ACM, 49(4):496-511, 2002. 1

[7] C.J. Ash: Finite semigroups with commuting idempotents. J. Austral. Math. Soc. (Series A), 43:81-90, 1987. 6

[8] A. Bertoni, C. Mereghetti, And B. Palano: Quantum computing: 1-way quantum finite automata. In DLT 2003, volume 2710 of Lecture Notes in Computer Science, pp. 1-20, 2003. 1

[9] A. Brodsky And N. Pippenger: Characterizations of 1-way quantum finite automata. SIAM Journal on Computing, 31(5):1456-1478, 2002. 1, 2, 3

[10] S. Eilenberg: Automata, Languages and Machines. Volume B. Academic Press, New York, 1976. $7,14,16$

[11] M. Golovkins And M. Kravtsev: Probabilistic reversible automata and quantum automata. In COCOON 2002, volume 2387 of Lecture Notes in Computer Science, pp. 574-583, 2002. 1 


\section{Marats Golovkins AND JEAN-ERIC PIN}

[12] M. Golovkins AND J.E. Pin: Varieties generated by certain models of reversible finite automata. In COCOON 2006, volume 4112 of Lecture Notes in Computer Science, pp. 83-93, 2006. 2

[13] A. Kondacs And J. Watrous: On the power of quantum finite state automata. In Proc. 38th FOCS, pp. 66-75, 1997. 1, 2

[14] S.W. MARGOLIS AND J.E. PIN: Inverse semigroups and varieties of finite semigroups. Journal of Algebra, 110:306-323, 1987. 6, 9

[15] C. Moore And J.P. CRutChFIELD: Quantum automata and quantum grammars. Theoretical Computer Science, 237(1-2):275-306, 2000. 1

[16] A. NAYAK: Optimal lower bounds for quantum automata and random access codes. In Proc. 40th FOCS, pp. 369-377, 1999. 1

[17] J.E. Pin: Varieties of Formal Langages. North Oxford, London and Plenum, New-York, 1986. 6, 13,14

[18] J.E. PIN: On the languages accepted by finite reversible automata. In ICALP 1987, volume 267 of Lecture Notes in Computer Science, pp. 237-249, 1987. 9, 11

[19] J.E. PIN: On reversible automata. In LATIN 1992, volume 583 of Lecture Notes in Computer Science, pp. 401-416, 1992. 9, 11

[20] J.E. PIN: Eilenberg's theorem for positive varieties of languages. Russian Mathematics (Iz. VUZ), 39(1):80-90, 1995. 6, 16

[21] J.E. Pin, H. Straubing, And D. ThÉRIEn: Small varieties of finite semigroups and extensions. J. Austral. Math. Soc. (Series A), 37:269-281, 1984. 11

[22] J.E. PIN AND P. WEIL: Semidirect products of ordered semigroups. Communications in Algebra, 30(1):149-169, 2002. 5, 6, 7, 11, 16

[23] J.E. PIN AND P. WEIL: The wreath product principle for ordered semigroups. Communications in Algebra, 30(12):5677-5713, 2002. 9, 11, 17

[24] L. PolÁK: Syntactic semiring of a language. In MFCS 2001, volume 2136 of Lecture Notes in Computer Science, pp. 611-620, 2001. 8

[25] G.B. Preston: Inverse semi-groups with minimal right ideals. J. London Math. Soc., 29:404-411, 1954. 7

[26] V.V. VAGneR: Generalized groups. Dokl. Akad. Nauk SSSR, 84(6):1119-1122, 1952. 7 


\section{AUTHORS}

\section{Marats Golovkins}

Faculty of Computing, University of Latvia,

Rainga bulv. 29, Riga LV-1459, Latvia

marats [at] latnet [dot] lv

Jean-Eric Pin

LIAFA, Université Paris Diderot and CNRS,

Case 7014, 2 Place Jussieu, 75205 Paris Cedex 13, France

Jean-Eric.Pin [at] liafa [dot] jussieu [dot] fr 